\title{
Solitons: A Cutting-Edge Scientific Proposal Explaining the Mechanisms of Acupuntural Action
}

\author{
Adrián Ángel Inchauspe 1,2,3,4* $^{*}$ \\ ${ }^{1}$ Scientific Department, Argentina Acupuncture Society, Buenos Aires, Argentina \\ ${ }^{2}$ Argentina Resuscitation Council, Buenos Aires, Argentina \\ ${ }^{3}$ Investigation Department, HINEA y C. “Dr. Alejandro Korn”, La Plata, Argentina \\ ${ }^{4}$ Medical Sciences Faculty of La Plata University, La Plata, Argentina \\ Email: *adrian.inchauspe@yahoo.com.ar
}

How to cite this paper: Inchauspe, A.Á. (2018) Solitons: A Cutting-Edge Scientific Proposal Explaining the Mechanisms of Acupuntural Action. Chinese Medicine, 9, 7-33.

https://doi.org/10.4236/cm.2018.91002

Received: January 18, 2018

Accepted: March 9, 2018

Published: March 12, 2018

Copyright $\odot 2018$ by author and Scientific Research Publishing Inc. This work is licensed under the Creative Commons Attribution International License (CC BY 4.0).

http://creativecommons.org/licenses/by/4.0/

(c) (i) Open Access

\begin{abstract}
The Theory of Morphic Fields explains how co-evolution through collective information fields acquires its own organization pattern, nurtured by habits or thoughts that "in-form" the memory of species. The influences of electromagnetic fields (EMFs) on bio-energy transport and its mechanism of changes has been investigated through analytic and numerical simulation as well as experimentation. As an answer to that need Therapeutic Acupunctural Resonance is born. This innovative therapeutic is rooted in concepts from classic Physics as well as Field Quantic Theory-highlighting the practical application of the criterion defined by the Soliton theory and the current, extraordinary discoveries at Cellular Quantic Kinetics level. The development of Therapeutic Acupuntural Resonance has taken into account to a certain extent these concepts: both the compatibility between the Five Element Theory and the principles of Euclidean Geometry, as well as the new forms of interpretation of Quantum mechanisms involved in Cellular Kinetics and its relationship with Chinese Ancient wisdom.
\end{abstract}

\section{Keywords}

Therapeutic Acupunctural Resonance, Morphic Fields, Soliton Theory, Cellular Quantic Kinetics

\section{Background}

“Qui ne sait pas cequ'ilcherche, ne comprendra pas cequ'iltrouve".

Claude Bernard (12/7/1813 - 10/2/1878) 
In the early 1990s, Rupert Sheldrake launched A New Science of Life: Hypothesis of a Formative Causation, stating the Theory of Morphic Fields. Based on the Inter-Dependency theory, Sheldrake explains how co-evolution through collective information fields (i.e. morphic fields) acquires its own organization pattern, nurtured by habits or thoughts that "in-form" the memory of species [1].

As an answer to that need Therapeutic Acupunctural Resonance is born. This innovative therapeutic is rooted in concepts from classic Physics as well as Field Quantic Theory-highlighting the practical application of the criterion defined by the Soliton theory and the current, extraordinary discoveries at Cellular Quantic Kinetics level [2] [3].

\section{Introduction}

\subsection{Concept of Morphogenetic Fields}

The general aim of a morphogenetic field-determined by the behavior in the development of a cell-shall depend on the instructional signals from its surrounding space: they presuppose the modular nature of embryo cells because of their genotype/phenotype relationship [4].

According to Gilbert, morphogenetic fields-not genes or cells-generate variations leading to the changes in biological evolution (Gilbert, 1996). Morphogenetic fields theoretically describe the intrinsic relationship existing between the biological information encoded in cells and the realization of the geometric form in the development of any organism [5].

The influences of electromagnetic fields (EMFs) on bio-energy transport and its mechanism of changes have been investigated through analytic and numerical simulation as well as experimentation. As such, EMFs can affect the structure of protein molecules and change the properties of the bio-energy transported in living systems residues [6].

The development of Therapeutic Acupuntural Resonance has taken into account to a certain extent these concepts: both the compatibility between the Five Element Theory and the principles of Euclidean Geometry, as well as the new forms of interpretation of Quantum mechanisms involved in Cellular Kinetics and its relationship with Chinese Ancient wisdom. Following Rupert Sheldrake and scientific concepts mentioned above, all of them converged in this modern view of what we now understand as Morphic Resonance [2] [3].

\subsection{The Concept of Bioplasma and Its Multiple Applications}

Bioplasma could be understood as an information generator and transformer and is constituted by protein semiconductors, piezoelectric compounds and molecules (melanin, neuromelanine, melatonin, DNA, RNA). It helps to maintain the symmetry of matter, integrating extensive environmental factors (changes in temperature, pressure, gravity, electric fields, magnetic, torsion, acoustic, optical and chemical changes) mutually interacting among interconnected fields of positively and negatively charges, thus preventing matter destabilization [7]. For 
this to happen, it is necessary to provide energy in various forms. As we will see later in this work, bio-energy transportation along protein molecules is curiously performed by the soliton phenomena caused by the dipole-dipole electric interactions between neighboring amino acid residues [6].

Understanding such successful integration is a fundamental step, for it requires a profound knowledge over the intrinsic mechanisms of the soliton. The recent progress and surge of interest in nonlinear equations has been impressive, at the same time, novel and interesting physical applications multiply in very widely different physical situations, as in the case of solitons.

The Advanced Study Institute of "Nonlinear Equations in Physics and Mathematics" (Istanbul, August 1977) provided a survey of certain recent developments in the theory of integrated dynamic systems consisting essentially in the display and analysis of a number of "body conditions" compatible with the soliton theory [8]. It is therefore not surprising that, for those systems having nonlinear configurations (such as our bodies), said solitons are characterized by remarkable mathematical properties that have been recently uncovered [8].

\section{Materials}

\subsection{Definition of Instrumental Mediator}

The Russian psychologist Lev Vygotsky (1896-1934) believed that man acts over reality adapting to it, transforming himself through his "mediators", conceptualized as "tools" (material resources) or "signs" (language, music), which set culturally determined and contextualized interactions in a socially cooperative environment [3].

Movement has an innate control mechanism and is slightly susceptible to modification by learning. Its apparent "plasticity" consists of the performance of a multitude of attitudes in response to the surrounding environment. Movement retains its symmetry determining the coordination of overall body mechanics [3] [9]. The disturbance of this symmetry is evident in mental dysfunctions. The interplay of learning movements along with the internal representation is essential when learning how we can recognize objects among its space [10].

All the sign systems - the group of phonetic, graphic, tactile, etc. instruments-are considered by Vygotsky as a great system of instrumental mediation [10] [11]. This concept can be perfectly adapted from psychology to biology, and then applied to pathological conditions of different natures through Musical Treatment.

Some of the following conditions identify an Instrumental Mediator, namely

- It is a technological support of information acting as an energy transductant.

- It is the carrier of an intentional message in order to cause an effect on the recipient.

- It implies the application of a kind of knowledge that is intentionally structured.

- It allows us to adopt specific symbolisms for learnt knowledge. 
- It organizes information at pedagogical and therapeutic levels as well.

It clarifies the significance of information and the construction of self-awareness both from our internal and from its surrounding environment conditioner [3] [10].

\subsection{A Brief History of the Soliton}

The phenomenon was first described by the Scottish engineer and naval designer John Scott Russell (1808-1882) while studying the propagation of water waves in shallow channels [12].

A simplified analysis from Physics would enable us to describe soliton as a Wave of Translation. This wave is made up of combination of sine waves at different frequencies which add up into a complex wave which remains undispersed, the waves keep their stability thanks to reciprocal nonlinear interactions coupled at a critical value [13].

Russell's original description about this "propagation of solitary waves" as a constant situation within the sphere of Hydrodynamics was only called soliton towards the mid-1960s. It implies a balance between nonlinear and dispersive effects, actually comparable to avant-garde modern branches of Physics [14] and Acupuncture's new physiological theories and therapeutic modalities.

In Nature there exist other kinds of similar phenomenon like the earthquakes and the tsunami-that involves maritime underwater currents-or the pororoca that involves rivers and can be found in the Orinoco and Amazon rivers; the Severn in the United Kingdom and also Fundy Bay in Nova Scotia [15].

The dissipative soliton concept is a fundamental version of solitons developed in conservative and integrable systems. Standard soliton theory began in the 1960s ideas from nonlinear dynamics theory proposed by Poincaré and Prigogine's studies for systems far from equilibrium. In this way, solitons constitute the framework of particular "integrable equations" that could be adapted to decipher "integrable systems" [16].

Nonlinear dynamics appears to be a feasible explanation of how our Universe evolved from chaos. To some extent, the Chinese scholars were already aware of the solitonic theory and I myself have been able to verify they have applied their principles from CTM to their masterful conception of the Universe [17].

\section{Methodological Approach}

\subsection{Analysis of the Kinetic, Hydrodynamic and Quantum Properties of the Soliton}

\subsubsection{A Scientific Definition of Soliton}

The term "soliton" is used in a generic sense to denote all examples of dynamic vibrational self-trapping (understanding here the stable pulse does not disperse). As stated earlier, solitons exemplify this peculiar phenomenon: a kind of pulse-like solution applicable in hydrodynamics, optics, plasma physics, solid state physics, biochemistry, molecular science, elementary particles theory or as- 
tronomy [17].

In Acupuncture, solitons seem to move as high-amplitude, nonlinear "solitary pulses" that compress body energy. In this respect, they resemble shock waves due to their "hydraulic jump" because they can modify the environment for other small neighboring waves for their large amplitude, and thus feed on them by attracting them into their vast potential waves [18]. This is what seems to happen while intercepting specific point along the meridians, be it through digital or acoustic stimulation of the needles.

These localized entities function as carriers of mass, electric charge, vibrational or electromagnetic energies, etc. depending on the particular non-equilibrium environment [18]. That could be mathematically justified by means of the paradigmatic model of nonlinear equation (Schrödinger's wave equation) [19].

\subsubsection{A Scientific Analysis of the Solitonic Wave}

Solitons represent solutions to the Korteweg-de Vries equation. Given a train of solitary waves, the speed of wave spacing is continuously variable. Solitons can undergo "collisions" in which their identity and coherence are preserved. Further on we shall insist on this point [20].

The solitonic wave can either be continuous of present like Gaussian pulses (i.e. generalized pulse), always made up of two main components: the sheaves and the carrier waves. The latter form a pack of waves that propagate undisturbed, keeping their nonlinear, longitudinal and coherent characteristic [21].

Dimensional solitons owe their existence and permanence to a balance of two forces:

- Balance between Dispersion/Diffraction effects: each wave frequency propagates at different speeds within the soliton sheath [19]. A consequence of that balance relationship could result in the Kerr effect, i.e. waves overtaking others due to propagation in a nonlinear environment, seeking to cause energy expansion.

- Non-linearity effect: seeks to compress solitons.

The compensation between these two effects keep the wave "package" intact, which paves the way for the manifestation of soliton [21].

A system that changes the shape of the wave creates anharmonic distortion. This is the main characteristic of nonlinear system: if it affects the wave symmetrically, the harmonics produced are all odd. If it affects the harmonics asymmetrically, at least one even harmonic is produced [22].

\subsection{Solitons and Music: Phonons}

\section{Phonons: A Definition}

A phonon is a quantum mechanical description of an elementary vibrational motion in which grating of atoms (or molecules) oscillates at a uniform frequency (mostly in a nonlinear environment). The concept was introduced in 1932 by Soviet physicist Igor Tamm and its name comes from the Greek word $\varphi \omega v \eta \dot{~(p h o n e)), ~ w h i c h ~ m e a n s ~ s o u n d ~ o r ~ v o i c e, ~ b e c a u s e ~ l o n g-w a v e l e n g t h ~ p h o n o n s ~}$ 
give rise to sound [23].

In Classic Physics this designates a normal mode of vibration; this means, a superposition of these elementary vibration modes (vide infra "Discussion"). While normal modes are wave-like phenomena in classical mechanics, phonons possess wave-particle related properties of quantum mechanics too.

In Quantum physics, a phonon is a collective excitation in a periodic and elastic arrangement of atoms or molecules in condensed matter, like solids and some liquids. Often designated a quasi-particle, it represents an excited state in the mechanical quantization of the vibration modes of interacting particles in elastic structures [23] [24]. Phonons play a major role in many of the physical properties of condensed matter, like thermo-electrical conductivity. For this matter, its study clearly resembles the bio-physical manifestations of human energy or $Q i$ [4].

Acoustic phonons are coherent movements of atoms of the lattice out of their equilibrium positions. If the displacement is in the direction of propagation, then in some areas the atoms will be closer, in others farther apart, as in a sound wave in air (hence the name acoustic [25]).

Displacement perpendicular to the propagation direction is comparable to waves in water. Acoustic phonons exhibit a linear relationship between frequency and phonon wave vector [26]. The frequencies of acoustic phonons tend to zero with longer wavelength, as might happen during puncturing stimulation in Therapeutic Acupunctural Resonance.

\section{Methodologica Approach}

\subsection{Creation of Solitons during Acupuncture According to Traditional Chinese Medicine}

According to Acupuncture treatises, the appearance of the "Qi arrival phenomenon" is key in acupunctural treatment: the longer it gets to appear at the appropriate location, the more uncertain results shall be.

However, a retrospective analysis from the avant-garde panorama offered by the soliton theory applied to Acupuncture might reveal other, more advanced, scientific motives. In cases of weakness of the patient's anti-pathogenic Qi (in turn, due to a general deficiency in Zhang Fu) which makes it difficult for the patient to reach that "propagated sensation", there is a number of basic manipulative maneuvers to provoke the desired reaction:

1) Withdraw and introduce the needle several times, this activates Qi by stimulus frequency.

2) Repeatedly rotate the needle in order to increase puncture stimulation.

3) Auxiliary stimulation methods. There are three additional methods to activate punctures:

- Scratch the tip of the needle with the nail;

- Flick the tip repeatedly

- Make the needle víbrate (see Figure 1) [27] 

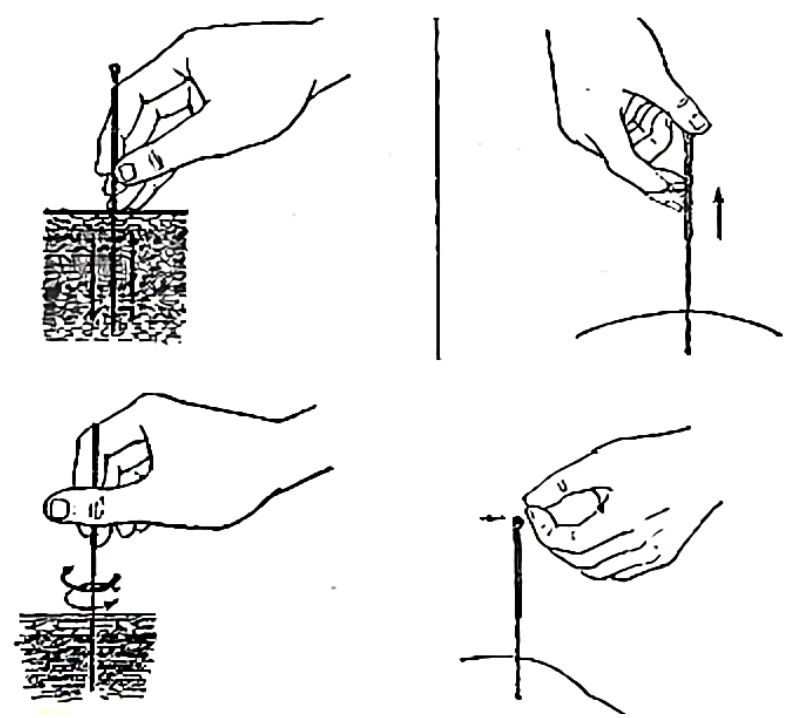

Figure 1. Creation of solitons during Acupuncture [27].

As stated in the last method, the Ancient Chinese masters were keen on provoking vibrations on the points in order to boost their therapeutical effects. Somehow, Therapeutic Acupunctural Resonance is in line with this methodological intention of the Pentatonic Scale (a natural follow up to the "Circle of Fifths" in Pythagoras' scale). These are supported by the subtle property of the phonons transported by those musical notes which are "holographically" charged with core information meant to recover the Qi in the meridian system. All this has a powerful influence on the nuclear genome [2] [3].

To combine the sounds corresponding to the Elements making up the Ancient Shu points-following a Generative sequence-enables the "re-generation" of solitonic frequencies inherent to each channel, restoring in this way the corresponding Qi flow at the energetic levels affected [2] [3].

\subsection{Tunnels of Solitons ("Cavity Solitons")}

A magnetic field can be conditioned like a soliton; precisely by "its property of digging "tunnels". For example, a superconducting metal is converted to soliton at a critical temperature-which in turn converts quoted metal into a superconductor [28]. However, if the magnetic field strengthens or expands its influence, solitonic magnetic vortices are created that penetrate the superconductor in the manner of a tunnel. According to Briggs and Peat,"... it is as if one soliton passes through the other" [29]. This explanation could lead to the reasons for the existence and abundance of the minute and superficial Acupuncture connective channels (Sun Luo and Fu Luo) located in the secondary vessels space (between the epidermis and the muscles) all along our bodies.

For Italian researchers L.A. Lugiato et al., the combined properties of spatial multi-stability observed for Cavity Solitons (CS) set the basis for several avant-garde applications which are being progressively validated within the $E u$ ropean STREP Project "Fun FACS" (Fundamentals, Functionalities and Appli- 
cations of Cavity Solitons) [30]. A particular consideration is that Cavity Solitons will transfer information content among physically distinct locations (as Acupuncture channels do) throughout the resonator's section.

The CS-carried signal propagates inside the resonator when one discriminates the speed of light in the medium and the speed of the CS when it drifts across the device [30]. Lugiato et al. observed that under the action of noise from $100 \mathrm{~mW}$ to less than $1 \mathrm{MHz}$ - that is capable to generate an "injection beam"-Cavity Solitons wander around until the distance between two of them becomes less than their interaction range, so that, eventually, they merge into a single and stable CS [19] [30] [31].

In fluids that do not create turbulence, solitons form cylinders and textures in such "superfluid" [32]. Either of these forms-both the "superconductor" and/or the "superfluid" - could perhaps be compatible with the description of the Acupuncture meridian's bio-dynamics: an energetic vibration energetic beam that-in a real or virtual way-traverse both parenchyma and stroma to rebalance our electromagnetic field.

In a kinematic viscosity medium comparable to the human stroma such travelling waves follow the Marangoni model for viscous flows. This is a crucial concept in the development of the Theory of Interfacial Turbulence, where long-wave instability due to "negative viscosity" or "active friction" is identified in the anisotropic dissipation-modified Boussinesq-Korteweg-De Vries equation for strongly dissipative flows [33].

\subsection{Applied Functions of Solitons}

\section{Solitons in the Human Body: Action Levels}

For Brizhnik, there exist at least three physical mechanisms of biological effects of magnetic fields can be identified, namely molecular, supramolecular and system, depending on at which of them such effects take place.

- Molecular mechanism: it involves effects of magnetic fields on ions, radicals, paramagnetic particles with unpaired electrons and spin, molecules, macromolecules, changing their states and reactivity.

- Supramolecular mechanism: it involves effects on membranes, mitochondria, microcrystalls, cell nuclei, etc.

- System mechanism: it is based on the synergetic effect of both molecular and supramolecular effects, which are processed by the corresponding system or by the whole organism and is manifested at biological levels (endocrine, nervous, etc.). The biological effects via this mechanism are more delayed in time, since they are result of the primary effects of the first two mechanisms [34].

The biological effects of weak static and oscillating magnetic fields are different and can affect mainly the nervous system. Thus, the recognition of magnetostatic changes, mainly low-frequency weak magnetic fields can cause physiological effects, influencing over any types of cells [34]. 
External signals (be them acoustic or visual) are converted into electric signals and then transmitted through neurons. Many of them are solitonic signals which represent a neuroactivity signal due to the "chaotic" superimposition of solitons [17] as we shall see further on in "Discussion".

Perhaps no other relationship between man and his environment has been scientifically more profitable than that which Russian physiologist I.P. Pávolv established. H. Pierre Klotz wrote about the deductions of the great Russian scientist:

"For the comfort of his experimental study, Pávlov strained himself in creating, at the 'tower of silence', isolated stimuli. This allowed for elementary connections and describe their laws; however, in fact, the complexity of the natural environment makes signs to act, in general, in groups or torrents". This seems to resemble closely the expression "chaotic superimposition" [35].

\subsection{Soliton Functions within Cells}

\subsubsection{Collagen and Solitons}

Collagen in a bio-macromolecule commonly present in living smooth tissues. Tropocollagen is conformed as an alpha-helix bio-polymer, with approx. $35 \%$ of glycine, $10 \%$ proline and $9 \%$ of hydroxyproline, with some traces of hydroxilisin strength [36].

For Pang Xiao fenget al. this molecule combines three alpha-helices assembled into a multi-helix tridimensional structure, where the hydrogen and covalent bonds appear in an alternant sequence, enhancing its tensile strength [37].

For Carieri\& col. [38], infra-red absortion of collagen shows at high-frequency rates (in range of $1000-4000 \mathrm{~cm}^{-1}$ ) that the amide-I vibration dominates the spectrum, which is also coincident with the appearance of excitations of solitons.

While Pang investigated-under high voltage-the influence of EMF over the collagen properties, solitons emerged in the infra-red spectrum of collagen (480 - $2000 \mathrm{~cm}^{-1}$ ). In this way, Pang Xiao Feng et al. were able to state that the influence of an externally applied electric field could justify solitons' bio-energetic transportation in collagen. This is done by means of the electrical properties amino-acids have from protein molecules [39].

From these studies it was concluded that the soliton induction of collagen can act as an optical fiber, causing other nonlinear effects as a waveguide mechanism acting over the fibers of collagen. Thus, through TCM practice, collagen fibers act as our own informative "net", generating an ultrafast communication transfer by means of Acupuncture all throughout the body.

\subsubsection{Collagen and Cellular Mechanical Transduction}

Referring previous idea to that of fascial membrane network in human tissues, such concept enables us to adapt the notion from André Thomas of muscular tone to that of cellular tone, which allows the introduction to the definition of Mechanical Transduction as a "property derived from Kinetics that cells possess when bearing constant tensions on their cytoskeleton" [2] [3] [9]. 
Such knowledge-clearly evident in physiopathology and therapeutics of the Tendino-muscular channels in Acupuncture-shows the property of the parenchyma of "encoding" within its cellular DNA the amplified or attenuated vibrations transmitted from stroma nets as a "sensation of arrival and propagation of energy" (known as T'chi phenomenon in Acupuncture). Albert Popp demonstrated that $1 \mathrm{~cm}^{3}$ of DNA can store as much information as in one trillion CD-ROMs [40]. In fact, as we will demonstrate later in this work, DNA could be conceived as a molecule for encoding bio-energy and the bio-information needed to exert biological function [41] [42].

Cytoskeletons in a cell have the ability to dynamically change the intracellular organization, by changing their network connections and information, but also to connect and reconfigure neighbouring cells. Its flexibility allows the cytoskeleton to intervene in a collective manner. Cytoskeletal processing of signals involves its microtubular structure assembling information similar to the formulation of a phonetic language [43] (see then "Instrumental mediators").

In this way, Kinematic Cellular Indetermination allows us to understand the role of sound stimuli which-travelling from the environment and through the Acupuncture needle to the connective fascia-reach and influence the expression of nuclear genome [2] [3] [44] [45].

\subsubsection{Solitons and Genetic Coding}

The genetic system of living organisms can be described as a triple unity of its structural and functional organization. It consists of holographic structures able to transfer data by means of electromagnetic and acoustic waves that form solitons. Recently science has made a major leap as regards this concept, paving the way for the theory of wave genome [17]. Besides, DNA functional development involves nonlinear pulse propagation as a real possibility; precisely through those cellular structures considered before. However, according with Alwyn Scott, we must first understand the linear equation for linear acoustic waves in DNA, because actual research over both optic and acoustic modes requires current comprehension of long-range forces in acoustic wave environment [18].

Up to Vladimir and Tijana Ivancevic [19], Yomosa considered the standard Watson-Crick double-helix B-form DNA model, in which conformation and stability of DNA and the polynucleotide double helices are determined by:

The $E B$ energy of the hydrogen $\mathrm{H}$-bonds between inter-strand complementary base pairs, given by

$$
E B=X n B\left[1-\cos \left(\theta n-\theta^{\prime} n-\pi\right)\right]
$$

where $B$ is a parameter associated with the H-bond energy, while $\theta n=\measuredangle(B n, P n)$ and $\theta^{\prime} n=\measuredangle\left(B^{\prime} n, P^{\prime} n\right)$ denote angles between horizontal projections of the complementary base pairs and their corresponding axes.

More recently, a Lagrangian field-theory based modeling approach to protein holding has been proposed including these terms: (i) nonlinear unfolding $\varphi 4$-protein at the initial state [19]. 
Again, all this previous development does not necessarily mean that everyone must fully understand these formulas; however, the aforementioned serves to make these works known and as a way of taking bibliographic record so that this work is clearly based and supported by scientific evidence.

Coinciding with previous concepts, another property derived from Physics called Cellular Transduction consists in the study of the transmission of data through signals that come from an external origin towards the interior of cells [44] [46].

According to both Gómez Yepes and Mc Clare, it is therefore possible that Mechanical Transduction can allow cells to "capture" energetic vibrations that in turn activate "tissue remodeling cascades". It also has been proved that molecules emit specific sound waves/frequencies that "trigger" cellular functional answers, activating the processing of data for intracellular cognition [47] [48] [49].

Nowadays, there exist mathematical patterns that allow us to understand the propagation of non-linear stimulations and their influence over the transcription of DNA. As we saw before, during such process, about 15 to 20 pairs are open making up a "transcription bubble" that is propagated as a "solitary wave" as represented by the Sine-Gordon nonlinear equations and Nash Game Theory [3] [19] [50].

\subsubsection{Current Concept of MIRNA (Micro RNA)}

"MIRNA" is an acronym identifying short sequences of ARN (micro ARN) [51]. Those short polyribonucleitids-barely 21 - 22 nucleotids-are the "transcription bubbles" [52] mentioned before which are capable of transcribing any nuclear cell information, thus assuming a key role in regulation and control of genetic coding functions [2] [3].

Following Peter Petrovich Gariaev, in fact, it is not precisely understandable yet MIRNA's action mechanism [53]. It might restrain the translation of ARNm (while transporting RNA messages) controlling several tactic options in the metabolism of important cellular bio-systems. One example of this is the tumor suppressing protein P53, also known as "genome guardian" [54] — which can be found in the short arm of chromosome 17-that has the property of putting a stop to the cellular cycle and activation the enzymatic repair of DNA in case of neoplastic mutation, thus leading to apoptosis [53]. The author also believes MIRNA could manifest its strategic management through gene transposition during those messages-thanks to the embryogenetic memory of nonlinear systems-thus enabling genetic code "decoding", in accordance with Lingvistiko-Volonovoj genetic principles [55].

The nonlinear Schrodinger equation seems adequate to describe how proteins obtain their energetic source on the bases of its molecular structure, according to experimental values obtained by infrared absorption ( 1 - 3 to 5 - 7 micron wavelength) that can be absorbed by the protein molecules in living systems. Said results are also associated with the bio-information and its relationship with health 
or illness conditions, thus justifying the nonlinear quantum theory to explain bio-energy transportation [56].

Those "transcription bubbles" mentioned before are made from more than 20 different kinds of individual aminoacids. Each of them, in turn, composed by an amino group $\left(\mathrm{NH}_{2}\right)$, a carboxyl group $(\mathrm{COOH})$, and a side or radical (R) group attached to the alpha-carbon atom [57]. As Pang clearly explained in his work, when two aminoacids join, they release one molecule of water and form a peptide bond. This chain forms a variety of three-dimensional conformations. Regarding this author, the three most important structural configurations are:

- The alpha helix

- The beta sheet

- The globular conformation[57]

In biochemistry, the amide-I vibration is an expected response to metabolic energy storage. Quoted vibration releases-by ATP hydrolysis-aprox. 10 $\mathrm{Kcal} / \mathrm{mole}$. According to Alwyn Scott, dipole-dipole interaction among vibrating and non-vibrating peptide groups is transmitted "...as the antennae of a radio system" [58] [59] —something for me perfectly comparable to needles' performance during Therapeutic Acupunctural Resonance [2] [3] requiring that initially localized energy to in an elapsed time of aprox. one picosecond.

The energetic supply for most protein functions depend on ATP hydrolysis. An ATP molecule binds to a special part of the protein molecule, releasing 0.43 $\mathrm{eV}$, supporting bio-energetically the DNA reduplication and other biological processes in living tissues.

$$
\mathrm{ATP}_{4}^{-}+\mathrm{H}_{2} \mathrm{O} \rightarrow \mathrm{ADP}_{3}^{-}+\mathrm{HPO}_{2}^{-}+\mathrm{H}^{+}+0.43 \mathrm{eV} \quad \text { [57] }
$$

Such energy acts through a non-equilibrium process provided by the amide-I vibration. Up to Pang, the distribution of such vibrational quantum reaches frequency levels about $0.205 \mathrm{eV}$-the half of the energy released during ATP hydrolysis. For Pang, this value is nearly constant among all proteins [57].

All these factors leads us to assume that the energy source provided by ATP hydrolysis might be stored in the amide-I vibration. Pang interpreted that a double dipole-dipole interaction is the responsible of the amide-I vibrational spreading to neighbor peptide groups [57], thus generating non-linear interactions adopting a soliton shape.

In this way, Pang's theory showed how solitons could travel along the hydrogen-bonded spines of the alpha-helix of protein molecular chains. In a living system, this wave function is the result of the summatory and coherence of state of each excitons and phonons within it. Quoted phenomenon has been demonstrated by Hamiltonian Davydov sequence, despite the shortness of the obtained wavelength [60].

If a certain amount of vibrational energy coming from the amide-I groups is located at one molecular end of the alpha helix, it will suffice-as we said before-a longitudinal sound to react in a sufficient degree to keep the soliton wave coherent [61]. For me, everything that is topographically described as 


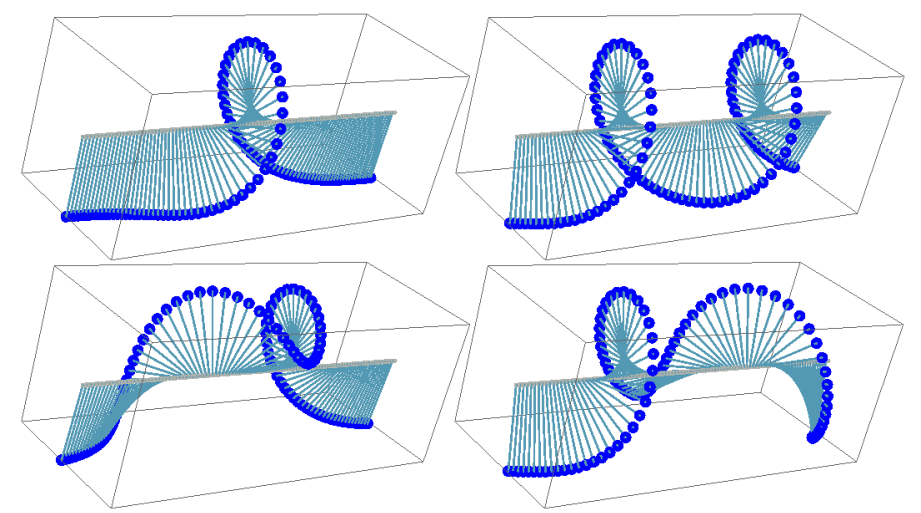

Figure 2. Sine-Gordon Solitons, Kinks and Breathers as Physical Models of Nonlinear Excitations in Living Cellular Structures [19].

"longitudinal" (length) is certainly compatible with the idea of meridian, and therefore of the Acupuncture map.

For Pang, such wave function is the sum of a product of quasi-coherent state of phonons, with a new soliton thermally stable, mathematically demonstrated in his work [61].

The energy is stored in complementary modes in the form of helical mass flow in the filaments and as local magnetic fields "...due to the paramagnetic behavior of the plasma in the filaments". It seems that solitons exit in the form of large-amplitude plasma waves. Hashimoto has shown theoretically many years ago that solitons can be expected to travel along ideal vortex filaments (see Figure 2) [61].

In his own words, Prof. Hashimoto expressed: "Theoreticians are now excited about the identity-and coherence-preserving properties of solitons in plasma. It was experimentally demonstrated twenty years ago that diamagnetic plasma vortices (plasmoids) [62] can bounce off each other like billiard balls. The plasma vortex is a tightly compressed coherent package of compressed energy" [63].

Thus, the cyclicality of quantum interactions controls the process of life because they link between optical, electrical, magnetic, mechanical phenomena and biological mass. Phonons combine all quantum processes with movement of mass, while photons constitute an information centre in cell transmission activity [64].

Incredible as it may seem, the Chinese Acupuncture was not alien to all this knowledge. As I explained in other works, the informative mission that proposes the contact of the needle with specific points of action on the organism tends to correct a certain biological function. The quasi-particles that constitute the phonons power and amplify said quantum information, transmitting it in harmonic tones previously ordered in a Cheng generative sequence [3].

\subsection{Non-Linear Quantum Dynamics of Ribosomes and DNA}

According to Peter Petrovich Gariaev, the previous quotes are closely related to an experiment carried out back in 1984-after an analysis by laser spectrosco- 
py-where in Russia the phenomenon of recurrent DNA memory was discovered [65]. The spectral compositions on DNA, ribosome and collagen preparations were analyzed, determining the vibratory functions of each of these samples on a sound field. This behavior in non-linear vibratory systems is known as the Fermi-Pasta-Ulam effect (FPU) in honor of the physicists who discovered this phenomenon (Enrico Fermi-Stanislav Ulam and J. Pasta) examining the vibrations of the metal using the Maniac I computer in 1956 [66].

When the metal received energy, the atoms vibrated; but their electrons remained in the "metal grid", vibrating in unison; that is, collectively, and producing a certain "note", possibly associated with a particular type of energy [67]. To observe the behavior of the energy as vibratory notes in the grid, Fermi, Pasta and Ulam curiously prepared a model of five musical notes (as it is used during the Therapeutic Acupunctural Resonance) looking for how they interacted with the grid. The nonlinear behavior of the experience transformed the grid into an ideal field for solitons: when a vibrational frequency lost energy, the solitonic interconnections allowed another to begin to gain it from the others, agglomerating successively in each modality [66].

Previously quoted data reminds us of the implicit purpose in a prefixed combination of points to treat certain nosological condition by Acupuncture: the vibratory frequency achieved by the excitation of nonlinear solitonic interconnections also ensures the dynamic energy stability of biological systems thanks to this new modality of therapeutic combination [2] [3].

A perfectly adaptable model to demonstrate the action of Therapeutic Acupuntural Resonance and its Mechanical Transduction through solitons can be found in the investigations of Yamile Cruz and Ramón Fayad, published in the journal of the Faculty of Medicine of Bogotá [68].

According to Cruz and Fayad, non-linear models can be constructed that justify the transfer of wave signals by the excess energy that is released in the hydrolysis of GTP (guanidin-triphosphate) after the tubulin assembly of the microtubules. Approaches from classical Physics to the transport of signals in microtubules assume that the dimers can be in $\alpha$ or $\beta$ conformation, in turn influenced by their neighboring dimers (in the same way that we saw above how neighboring waves are recruited in the integral constitution of a soliton). For Cruz and Fayad, these piezoelectric properties of microtubules suggest new theoretical schemes about the internal structure of axons and their functional role in synaptic communication [69].

In reference to the work of Pang, his results were introduced as a suitable model for studying the vibrational spectra of protein molecules with a simple computational program [70]. Infrared vibrational modes of amide-I are equivalent with that of the infrared lights according to the resonance conditions of Physics, showing peculiar soliton-induced patterns [71].

Being immersed within the cytoplasm-interacting with both their ionic electric forces and the viscous force that dampens their displacement-microtubules 
must necessarily be included in the equation for this model of transportation of cellular solitonic waves (see Figure 3).

For this reason, the authors Yamile Cruz and Ramón Fayad illustrated us with the following deductions emanated from the equation presented above: "Under these considerations, in the following formula the dynamics of the dipoles in a $M T$ is where $u_{n}$ represents the projection of the longitudinal displacement of the $n$-th dimer on all the protofilament, $N$ is the number of dimers and $M$ is the mass of each of them. The first term is the kinetic energy, the second the elastic energy given by the vibrations of the nearby dimers, the third and fourth terms represent the anharmonic potential and the last- $q E u_{n}$ is the electrical energy due to the electric field external to the MT. The quantities $A$ and $B$ are constants, $E$ is the field and $q$ is the moving charge in the protofilament" [68] [69].

In some way, we must strive to make this cutting-edge knowledge-derived from Exact Sciences-compatible with those patterns that regulate the Chinese Medical practice. In fact, all the aforementioned studies and investigations are useful to verify the underlying precision implicit in the Ancient Chinese postulates, granting their well-deserved validity, moving away from the concept of an empirical or currently obsolete knowledge.

\subsection{Theory of Solitons in the Nervous Transmission}

\subsubsection{Experiments with the Giant Squid Axon}

In 1938, Alan Lloyd Hodgkin (1914-1998) as Junior Research Fellow, and Andrew Fielding Huxley (1917-) as a student, began studying the transmission of action potentials at the Plymouth Marine Biology Laboratory in Great Britain. Their first work was published in Nature, but three weeks later World War II broke out and investigations were postponed until the end of the war (their competitors, among them I. Tasaki, continued their investigations in Japan and Germany). His knowledge of Physics and Mathematics enabled Hodgkin to contribute to the development of the radar, a secret project of the British government. Other projects dedicated to the microwaves Physics as the radar and other equipment used in aviation, were interspersed with experiments on action potentials. Huxley had not been able to continue his studies because of the interruption of classes; but at the end of the war, Hodgkin and Huxley restarted their work with the axons (see Figure 4) [72].

Two discoveries were decisive for the task of Hodgkin and Huxley: the electronic equipment and the particular anatomy of the squid. In 1946 Cole and Marmont had developed the equipment for the control of cell membrane potential (voltage-clamp). Hodgkin already knew it for having visited Cole's laboratories

$$
H=\sum_{n}^{N}=1\left[\frac{1}{2} M\left(\frac{d u_{n}}{d t}\right)^{2}\right]+\frac{1}{2} K\left(u_{n+1}-u_{n}\right)^{2}-\frac{A}{2} u_{n}^{2}+\frac{B}{4} u_{n}^{4}-q E u_{n}
$$

Figure 3. Equation for this transport model of solitonic cell waves published in Cruz \& Fayad research [69]. 


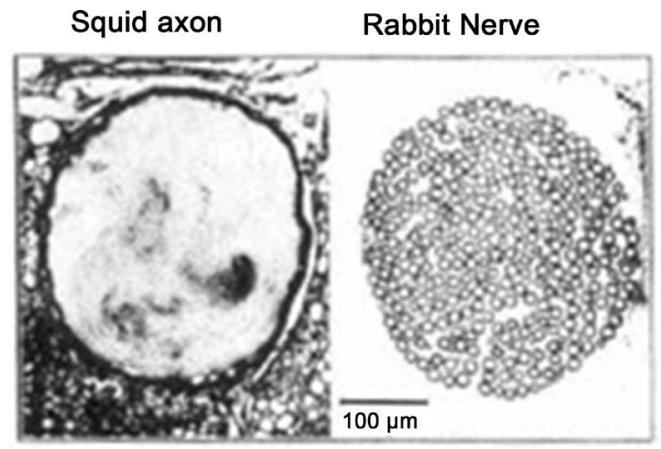

Figure 4. Microphotographs of a Loligo pealeii $s$ giant squid axon and a rabbit nerve composed of numerous axons [73].

and other researchers in the United States. The voltage-clamp allowed to maintain the membrane potential when the cell was stimulated, avoiding the explosive phenomenon- "all or nothing" reaction-of the action potential [73] [74].

The electric current that was injected so that the potential remained at a set value was similar in magnitude and opposite in sign to the current carried by the ions that pass through the membrane. The other key element to his task was the giant squid axon [75].

This rare anatomical peculiarity and its function were discovered in the early 1930s by the British zoologist J. Young (1900-1997). Squids are mollusks with ten tentacles and a tubular body innervated by axons of large diameter that in some species reach about $1 \mathrm{~mm}$, an enormous dimension when compared with 10-20 $\mu \mathrm{m}$ of mammalian axons [72].

This same principle was used when considering the experiments with sounds carried out to verify the stimulation of the needles during Therapeutic Acupunctural Resonance. The attempt to magnify the phenomenon could make visible situations that, although we know they actually exist, it would be almost impossible to quantify. If someone shouts against a wall, we surely know that phonons will pass through it until they reach our ears; but there are still no apparatuses that can register the deformation of said wall when the sounds pass through it. In the same way that microbiologists use the microscope to distinguish extremely small images to be appreciated by the human eye, choosing longer needles and intensifying the sound stimulus of the musical notes have made it possible to register changes that would otherwise go unnoticed by our senses.

These results-which will include conclusions of the Department of Neurosciences and the Commission of Scientific Research of the Province of Buenos Aires-will be published in another specific chapter for this purpose in this same Editorial, so as not to take up the valuable pages of this journal in order to edit other new transcendent works.

According to Hodgkin, quoted work of 1962 was the one that convinced the Nobel Prize Committee about the hypothesis presented in their investigations. Hodgkin and Huxley were awarded the 1963 Nobel Prize; John C. Eccles 
(1903-1997) shared it with them for their work on the synapses of the central nervous system [76].

The works of Hodgkin and Huxley anticipated several phenomena. The separated existence of sodium and potassium channels, their kinetics and tetrameric structure and the presence of a charged particle in the thickness of the membrane that-when moving according to the membrane potential-would be responsible for the activation of ion channels [77] [78].

These two British researchers were able to demonstrate that the nerve impulse traveled from the body of the neuron to the end of the axon, which allowed the brain to coordinate all the activities of the organism. With this discovery they were able to understand the solitonic propagation of signals along the axon of the neurons. The contribution to the advancements of knowledge in this field by the three scientists profoundly influenced the research for a wide range of neurological disorders [78]. Eric R. Kandel, a Nobel laureate in 2000, said that the work of Hodgkin and Huxley "made the cell biology of neurons what the structure of DNA did for the rest of general biology" [79].

\subsubsection{Solitons and Their Influence in Anesthesia and Neurosciences}

The work of Huxley and Hodgkin allowed us to explain how anesthetic physiology works, something that challenged the Neuroscience knowledge of that time. This justifies how some anesthetics block the movements of the ions, so the painful information does not reach the upper areas of the brain. The electric conduction by the nerves was also the base for bionic prostheses or innovative devices that move with electrical impulses and allow a progressive recovery of the functionality of lost limbs. It also made it possible to identify some genetic diseases called channelopathies, which arise due to a defect in the ion channels.

Xiaofeng Pang et al. studied and revealed this mechanism, numerically simulating the features of the movement of solitons along protein molecules with both, a single chain and with three channels by using the Runge-Kutta method and Pang's soliton model under the action of electro-magnetic fields with the strengths of $25,500,51,000,76,500$, and $102,000 \mathrm{~V} / \mathrm{m}$ in the single-chain protein, as well as $17,000,25,500$, and $34,000 \mathrm{~V} / \mathrm{m}$ in the three-chain protein, respectively. Results indicate that the soliton disperses at $102,000 \mathrm{~V} / \mathrm{m}$ in a single-chain protein and at 25,500 and $34,000 \mathrm{~V} / \mathrm{m}$ in three-chain proteins. Lastly, we experimentally confirm the existence of a soliton and the validity of our conclusion by using the infrared spectra of absorption of the collagen [70] [71].

Thus, we can affirm that both the described neural mechanism and the corresponding theory of bio-energetic transporting data in living systems have serious, certain and demonstrable biological effects.

After an extensive mathematical procedure in the continuous limit, we will arrive, as we quoted before, to one of the expressions of the Gordon equation, whose solutions result in soliton waves that propagate along the microtubule at a speed of estimated propagation approx. at $10-2 \mathrm{~m} / \mathrm{s}$ [80]. 


\section{Discussion}

\section{Notions of "Continuous Limit"}

From the theory of electromagnetism we know that the components of an electromagnetic field are influenced by Maxwell-Duffing equations. In many cases, the magnetic component of an oscillating electromagnetic field is weaker than its electric component. Nevertheless, quoted magnetic component becomes the main cause which affects biological systems. This is understandable because living tissues are composed mainly of water, which significantly absorbs electrical radiation, allowing its magnetic component to penetrate the stroma, thus affecting it locally and deeply inside. Quoted external stimuli originated at skin level-where Acupuncture Chinese Sages localized its biologically active points-to establish a close interaction of these electrical components as coupled among in an oscillating electromagnetic field [3] [34].

A continuous field can be defined as a group of oscillators coupled among themselves, giving an idea of elastic strength. Macroscopically speaking, the system is defined by the tension in each of its points. The Integrated Tension of those points represents the continuous fields [81].

By giving the idea of coupling the oscillators, it will be easier to represent the vibrations of atoms of a solid as a "superposition of its normal frequencies". Considering this phenomenon in a continuous matter, the density or tension of a solid would result in a "superposition of plane waves" of each of its components. Each harmonic mode of the group of oscillations would correspond to a certain plane wave-but added to its continuous limit.

The giant axon model of the squid allowed Hodgkin and Huxley to demonstrate neuronal physiology in an amplified manner. In the same way, experiments carried out by CONICET and CIC (branches of the Argentinian Scientific Research Comission) and by Dr. José Santisteban sought to magnify-with very long needles and lower musical tones-this phenomenon called "Neurosensitive Transportation" in order to make evident what, at first glance, was unviable to appreciate. As it was explained before, if a train is coming towards a station near our town, we can hear its approach by supporting an ear on the tracks, even if it is impossible to see the vibration in the rail. Thus, the magnification of the effect of sound on the needles made it possible to visualize how the punctures are driving their vibrations through it [3] [4].

In 1971, the Russian scientist A.S. Davydov proposed the soliton theory t o explain the transport of "energy packages" in very long helical molecules, such as amino acids [60]. According to this theory, bio-energy transports solitons along the helical protein molecules by the "stretching vibration" of amide residues affected by energy released from ATP hydrolysis, which causes a vibrational quanta called excitons while the deformation of amino acid residues also occur simultaneously [71].

Thus, the nonlinear coupling interaction between excitons and the deformation of amino acid residues that occur in this process, make such excitons to be 
"self-trapped" as a soliton which is able to transport further distances along the protein molecules in virtue of the dipole-dipole interaction between neighboring aminoacid residues [56].

Thus, Davidov suggested that-having reached a certain threshold of energy-the helicoid of the molecule carried "vibrational packages" at more than one thousand meters per second, thanks to the principle of non-linearity applied to biological processes [60] [71].

This is a well-known soliton mechanism of energy transport in life systems. Davydov's theory works in helix protein molecules with three channels. In this case, a biological process of energy transport always occurs from the produced place to the absorbed place in the living system, which is carried out by means of protein molecules such as collagen, myosin, myoglobin, and actin [60].

There is a simultaneous resonant and strain reaction generating a collective interaction directed along the axis of the spiral. The resonance excitation jumping from one peptide group to another can be represented as an exciton, the local molecule strain caused by the static effect of excitation as a phonon and the resultant collective interaction as a soliton. Davydov's own model of muscular solitons was given by the nonlinear Schrodinger equation (NLS) [60] [71].

This vibrational dynamics transmitted from the DNA through the MIRNA, its ribosomes and collagen could explain the nature of the transmission properties of the nervous system, allowing the remote diffusion of its impulses. Even Peter Petrovich Garyaev is allowed to assume that this transcription of DNA would be responsible for tissue regeneration, acting on the memory of biosystems on morphogenetic physical fields [65].

Probably, it can be assumed that the micro RNA is a complex system of genetic texts with new ranges of meanings, in the manner of a "Universal Esperanto", as Gariaev said. According to this text, the MIRNA-coming from the DNA and RNA present in the original nucleus-would play the role of "quantum biocomputer" that enables the precise execution of the orders generated from the cell nucleus [65].

Many biological functions such as cooperative effects, allosteric transition, enzyme kinetics or drug intercalation within DNA found their dynamic mechanism through solitary waves (solitons) conducted by cell micro-tubules. This vibration pulse travels within its wave packet, thus maintaining its speed, shape and coherence [82]. According to Lin and Lapointe [83], the relationship between low-frequency phonons and their excitement potential over bio-macromolecules represents a scientific breakthrough which will allow us to be able to understand crucial changes both in biophysics and biomedicine [84] [85].

This finding indicates that the energy cannot be damped and dissipated in the transport process and that it is significant for biological processes because energy is retained in the transport process due to the feature and essence of the soliton. Thus, these life activities clearly require the indispensable presence of solitons. 
After having compared the concepts of Modern Physics with those of Traditional Chinese Medicine, we are in a position to observe a great number of clear coincidences between Eastern and Western knowledge [2] [3].

Using music as an Instrumental Mediator is an alternative modality of physio-pedagogic as well as therapeutic mediation.

The Instrumental Mediator enables the transposition of knowledge, providing possibilities to restructure new neuro-cognitive pathways, thus enabling alternative ways that propose new paradigms of self-learning. In this way, these mediators of knowledge establish a complex network that systematically contains, in its structure, all the new information [3] [11].

Therefore, the comparison of the phenomenon of Therapeutic Acupunctural Resonance through the notion of soliton seems to be adequately correct. Russell's description during his observation is compatible with the nomenclature that identifies the Old Shu points that carry the pathway of energy (Qi) through the main meridians of Acupuncture [2] [3] [86] [87].

In fact, their sequence clearly illustrates the increase of the Qi channel during its pathway through said points: that is why they are so called "Bubbling Well", "Spring", "Stream", "River" and "Sea" Points, consequently causing an impressive increase of flow due to their own stimulation [2] [3] [88].

Likewise, solitons would explain the displacement of signals during nerve transmission. This was previously interpreted as a telegraphic or telephone message, as an electrical impulse traveling on a cable. Today we know that these impulses do not even match the speed of light, but when reaching a critical level of energy, they travel in a solitonic way, without deforming or dissipating at a constant speed that does not exceed $70 \mathrm{~m} / \mathrm{sec}$. despite observing a latency period before enabling another stimulus [89].

It seems that the neurons retain a specific sensitivity to previously transmitted messages, involving in some way, a "quantum memory". In this way, solitons transmit data through the vibrations in the biological systems, as it happens with nervous impulses (neurosolitons); and they seem to be "holographically" filed in the neurons of the nervous central system after being transported up to there by the peripheral nervous system. This kind of "holistic memory" for neural messages could explain mechanisms that are consistent with brain metabolism thanks to the "interaction" and "intersection" of solitons [2] [3] [90].

Regarding quoted deductions, in his RNA-polimerase experiment Fröhlich strongly insisted that both higher and lower frequencies may be involved in said process (as it happens in long DNA-protein complexes), as the results obtained in such studies demonstrate that both Davydov and Fröhlich original theories can match in some point, helping to configurate a more complete panorama about energy storage and stimuli transport among biological molecules [60] [71].

Chinese music was born as a pentatonic anhemitonic scale, from its integrated location within the Five Elements; and Pythagoras determined by his harmonic 
means and the Circle of Fifths their influence and harmonization on the meridians representing those Movements [2] [3] [9].

A simple way to incorporate the ideal frequency rhythm for each channel or to correct their unbalanced situation is to insert through the needles-by way of "antennae"-capturing said frequencies, restructuring the affected meridian and through it, the Element it belongs to [3].

Morphic Resonance, therefore, finds effective application when pins are used as "resonators" which put the frequency of the Element pertaining to the channel in line, so that they transmit by means of punctures, pure vibratory patterns which shall recondition the Qi flow frequency which has been altered by the Chinese syndrome to be treated [80] [81].

According to Hameroff, synapses and neurons have a dynamic and complex structure that transmits their stimuli as biocomputers, acting through cell microfilaments and microtubules. Hameroff believes that microfilaments and microtubules, along the entire cytoskeleton incorporate the modules that have to assess the inherent nature of the hierarchy information [90]. According to Hameroff and Penrose microtubules and acting cytoskeleton function as the microprocessor and must be considered as biocomputer phones [91].

Cytoskeletons in a cell have the ability to dynamically change and reconfigure the cytologic organization through information of their network intracellular connections and with neighbouring cells, dividing its resources in a collective manner [42]. This kind of "intra-extracellular net" is able to reach an integrative collective processing of information at the molecular level, performing the function of a computer cluster [64] [91].

In terms of the Physics of the condensed matter, the description of the atomic oscillations of a solid as quasi-particles called phonons, presents theoretical evidence about the possible action of sound over the Therapeutic Acupunctural Resonance channels through Quantum Physics.

\section{Conclusions}

We have already seen intrincate peculiar connections between the Musical Major Pentatonic anhemitonic scale-typical of Chinese music-and the properties that Zou Yen attributed to the Five Elements coming from Chinese Cosmology [2] [3].

This way of interpreting the musical representations followed by TCM allows us to introduce substantial changes in its therapeutic methodology by the proposition of Instrumental Mediators. Under this new concept-and according to engineer Roberto Tambornino-the musical notes would act in such a way as to cause interaction between the patient's perception and its own nervous system [10] [11].

Vygotsky thought that human maturity implies a process of cultural advancement, being man's activity the motor of human development. The activity proposed by quoted author is one culturally determined by "mediators", since 
the social world is essentially formed by symbolic processes. Therefore, authors Amelia Alvarez and Pablo del Rio wrote in their work "Higher Psychological Processes in the Perspective of the Socio-Cognitive Theory" that all the sign systems-phonetic, graphic, tactile, etc.-are considered as a great system of instrumental mediation [10] [11].

For this reason, authors like Adam Adamski deduced that these people are dysfunctional as they appear to have "double meanings messages" that generate inappropriate movements, shortness of breath, lack of visual-motor coordination etc [64]. For Adamski, future investigations will move in the direction of the cognitive structures for energetic information, recognizing our body as a quantum data generator. Thus, electromagnetic, sound, spin or solitonic expressions of energy and bioplasma represent current innovative ideas that support next research explanations and results.

As mentioned at the beginning of this work in its "Background", the term in-formation has many meanings: it not only could be interpreted as something that carries a message, but I may also refer to the ability to organize systems or maintain an organized state [64].

Perhaps some patterns achieved by patients while being treated with Therapeutic Acupunctural Resonance may enable them not only to recover lost body functions, but also to re-learn them. This possibility of sequenced energetic re-configuration may allow human beings to re-establish new energetic circuits, empowering their capacity of biological regeneration and recovery [92]. Only in this way will we be able to understand that that Generative Sequence proposed by Zou Yen more than two thousand years ago, encircles within its cycle, a possible "resetting" of the functions lost due to the impairment resulting from degenerative processes [2] [3] [86] [87].

The most important conclusion is that Traditional Chinese Medicine (TCM) is far from being a pre-scientific empirical knowledge that cannot justify academically its pathophysiological mechanisms, as it is generally held by Western doctors. TCM is based on Exact Sciences concepts, since it has been developed in close relation with principles rooted in Greek mathematics and geometry. Most of its rules-compiled in apparent simple metaphors to facilitate understanding by its people-also include vanguard theories pertaining to Quantum Physics and Electromagnetic Field Theory.

I-Ching, as Lavoisier's Law of Energy Transformation, guides us to understand the changes produced at physiological as well as at structural level which occur within a biological system, as advocated for millennia by Traditional Chinese Medicine. Such morphogenetic changes constitute an example of the intrinsic dynamic balance between biological coded information within cells and the resulting Geometry in the structure and development of organisms [1] [2] [3].

Beyond the esoteric, philosophical and metaphysical questions implicit in the practice of Chinese Medicine, there are also physical-mathematical evidences, 
morphogenetic studies and the Quantum Theory of Fields that support this millenary knowledge. I hope to have fulfilled my intention to shed light on the avant-garde scientific foundations that justify the current validity of this Ancestral Healing Art.

\section{References}

[1] Giacobone, M. (2009) Morphic Resonance: A New Approach from Biology. (In Spanish)

http://budacuantico.blogspot.com.ar/2009/12/la-resonancia-morfica-un-nuevo-enfo que.html

[2] Inchauspe, A.Á. (2015) Therapeutic Acupunctural Resonance: The Original Research. Chinese Medicine, 6, 214-233. https://doi.org/10.4236/cm.2015.64024

[3] Inchauspe, A.A. (2016) Therapeutic Acupunctural Resonance II: New Discoveries That Justify the Outcomes of This New Therapeutic Modality. Journal of Biosciences and Medicines, 4, 39-45. https://doi.org/10.4236/jbm.2016.46006

[4] Morphogenetic Field (Developmental Biology). (In Spanish) https://es.wikipedia.org/wiki/Campo_morfogen\%C3\%A9tico_(biolog\%C3\%ADa_d el_desarrollo)

[5] Morozova, N. and Shubin, M. (2012) The Geometry of Morphogenesis and the Morphogenetic Field Concept.

[6] Pang, X.F., Chen, S., Wang, X. and Zhong, L. (2016) Influences of Electromagnetic Energy on Bio-Energy Transport through Protein Molecules in Living Systems and Its Experimental Evidence. International Journal of Molecular Sciences, 17, 3. https://doi.org/10.3390/ijms17081130

[7] Adamski, A.G. (2011) Bioplasma Concept of Consciousness. NeuroQuantology, 4, 681-691.

[8] Barut, P. (1977) Nonlinear Equations in Physics and Mathematics. Proceedings of the NATO Advanced Study Institute, Istanbul, 1-13 August 1977, 143-181.

[9] Inchauspe, A. (2014) Is Traditional Chinese Medicine Definitely an Exact Science? Comparison between the Oriental Five Elements' Theory and Euclid Five Regular Polyhedrons postulates. 2 nd International Conference and Exhibition on Traditional \& Alternative Medicines, Beijing, 25-26 August 2014, 255-277. https://doi.org/10.4172/2327-5162.S1.006

[10] Folegotto, I. and Tambornino, R. (2001) Instrumental Mediators. 16th International Symposium of Informatics in Education. (In Spanish)

[11] http://todoeducativo.overblog.es/pages/VYGOTSKY-5471284.html

[12] Russell, J.S. (1844) Report on Waves. 14th Meeting of the British Association for the Advancement of Science, York, September 1844 (London 1845), Plates XLVII-LVII, 90-311.

[13] Briggs, J. and Peat, F.D. (1994) Espejo y Reflejo: Del caos al orden. Editorial Gedisa, Segunda Edición, Barcelona, Chapter 4, 119.

[14] Russel, J.S. http://www.macs.hw.ac.uk/ chris/scott_russell.html

[15] Briggs, J. and Peat, F.D. (1994) Espejo y Reflejo: Del caos al orden. Editorial Gedisa, Segunda Edición, Barcelona, Chapter 4, 120.

[16] Akhmediev, N. and Ankiewicz, A. (2008) Dissipative Solitons: From Optics to Biology and Medicine. Lecture Notes in Physics 751, Springer, Berlin Heidelberg.

[17] Naumkin, P. (2008) What Is a Soliton? UNAM Bulletin No. 19, Mathematical In- 
stitute, Morelia-Campus, National Autonomous University of Mexico, Mexico City, 1-4.

[18] Christiansen, L. and Scott, A.C. (1990) Davydov's Soliton Revisited: Self-Trapping of Vibrational Energy in Proteins. Nato Science Series B Vol. 243, Springer Science + Business Media, New York, 31.

[19] Ivanisevic, V.G. and Ivancevic, T.T. (2013) Sine-Gordon Solitons, Kinks and Breathers as Physical Models of Nonlinear Excitations in Living Cellular Structures. Journal of Geometry and Symmetry in Physics, 31, 5. https://doi.org/10.7546/jgsp-31-2013-1-56

[20] Scientific Analysis of the Solitonic Wave. Source: Energy Storage: Compression and Switching, Book 84, 80, Code BOS 42.

[21] Soliton's Wave Properties. https://www.tuchemnitz.de/physik/KSND/abb/node6.html

[22] Harmonic Oscillators Operators. http://wiki.physics.fsu.edu/wiki/index.php/Harmonic_Oscillator_Spectrum_and_Ei genstates

[23] Phonons. https://en.wikipedia.org/wiki/Phonon

[24] Superposition of Elementary Vibration Modes. https://www.google.com.ar/search?tbm=isch\&sa=1\&ei=ctUdWvxE4muwgSmvrVw \&q=superposition+of+vibratory+waves+images\&oq

[25] Acoustic Phonons. http://ifit.mccode.org/Models_Phonons.html

[26] Maris, H.J. and Tamura, S. (2011) Propagation of Acoustic Phonon Solitons in Nonmetallic Crystals. Physical Review B, 84, Article ID: 024301.

[27] Creation of Solitons during Acupuncture. (In Spanish) https://es.slideshare.net/BarbaraFuentes3/tratado-de-acupuntura-iii-terapia-acupun tural-y-localizacion-y-combinacion-de-puntos-jose-luis-padilla-libro-completo

[28] Briggs, J. and Peat, F.D. (1994) Espejo y Reflejo: Del caos al orden. Editorial Gedisa, Segunda Edición, Barcelona, Chapter 4, 120.

[29] Briggs, J. and Peat, F.D. (1994) Espejo y Reflejo: Del caos al orden. Editorial Gedisa, Segunda Edición, Barcelona, Chapter 4, 123.

[30] Lugiato, L.A. CNISM and INFM-CNR, Dipartimento di Fisica e Matematica, Universit'adell'Insubria, ViaValleggio, 11, 22100 Como, Italy.

[31] Breathing Optical Solitons. https://laserlab.blogs.auckland.ac.nz/research/temporal-cavity-solitons/

[32] Nagasawa, T. and Nishida, Y. (1981) Experiments on the Ion-Acoustic Cylindrical Solitons. Plasma Physics, 23, 575-595.

[33] Briggs, J. and Peat, F.D. (1994) Espejo y Reflejo: Del caos al orden. Editorial Gedisa, Segunda Edición, Barcelona, Chapter 4, 122.

[34] Brizhik, L. (2014) Biological Effects of Pulsating Magnetic Fields: Role of Solitons.

[35] Klotz, H.P. (1957) El Aporte de Pávlov al desarrollo de la Medicina. Psique, Buenos Aires, 25. (In Spanish)

[36] Pang, X.F., Chen, S., Wang, X. and Zhong, L. (2016) Influences of Electromagnetic Energy on Bio-Energy Transport through Protein Molecules in Living Systems and Its Experimental Evidence. International Journal of Molecular Sciences, 17, 17. https://doi.org/10.3390/ijms17081130

[37] Pang, X.F. (1999) Influence of the Soliton in Anharmonic Molecular Crystals with Temperature on Mössbauer Effect. The European Physical Journal B-Condensed 
Matter and Complex Systems, 10, 415-428. https://doi.org/10.1007/s100510050871

[38] Carieri, G., Buontempo, V., Galluzi, F., Scott, A.C., Gratton, E. and Shyemusunder, G. (1984) Spectroscopic Evidence for Davydov-Like Solitons in Acetanilide. Physical Review B, 30, 4689-4698.

[39] Pang, X. (2000) Vibrational Energy-Spectra of Protein Molecules and Non-Thermally Biological Effect of Infra-Red Light. Institute of High-Energy Electronics, University Electronic Science and Technology of China, Chinese Academy of Sciences.

[40] Hasegawa, A. and Tappert, F. (1973) Transmission of Stationary Nonlinear Optical Pulses in Dispersive Dielectric Fibers. I. Anomalous Dispersion. Applied Physics Letters, 23, 142-144. https://doi.org/10.1063/1.1654836

[41] Cytoskeletal Processing of Signals Involving Microtubular Structure. http://dev.biologists.org/content/137/9/1407

[42] Microtubular Structure Assembling Information.

[43] Adamski, A.G. (2016) The Importance of Movement, Solitons and Coherent Light in the Development of Mental Processes. Journal of Advanced Neuroscience Research, 3, 25.

[44] Hameroff, S., Scott, H. and Tuszynski, J. (2002) Quantum Computation in Brain Microtubules? Decoherence and Biological Feasibility. Physical Reviews E, 65, Article ID: 061901.

[45] Hameroff, St., Rasmussen, S., Karampurwala, H., Vaidyanath, R. and Jensen, K. (1990) Computational Connectionism within Neurons: A Model of Cytoskeletal Automata Subserving Neural Networks. Physica D, 42, 428-449.

[46] Gómez Yepes, A. Why Low Frequency Electromagnetic Stimulation Produced by Inductive Magnetic Stimulator (EIMA in Spanish) Works Well in the Treatment of Various Diseases Including Autoimmune Ones? Coatepec, México, 2-7. http://www.terapiasmetabolicas.com/eima/

[47] Mulvany, M.J. (2000) Small Artery Remodeling in Hypertension. Current Hypertension Reports, 4, 49-55.

[48] Cardenas, M.L. (1991) Are the Transistors Enzyme-Complexes Found in Vitro also Transistors in Vivo? If So, Are They Physiologically Important. Journal of Theoretical Biology, 52, 111-113.

[49] Bartlett, P.N. and Prat, F.E. (1993) Modelling of Processes in Enzyme Electrodes. Biosensors Bioelectronics, 8-9, 451-462.

[50] Liberman, E.A. (1996) Cell Molecular Computers and Biological Information as the Foundation of Nature's Laws. Biosystems, 38, 173-177.

[51] Micro-RNA. http://ru.wikipedia.org/wiki/İèêðîĐí̂̂

[52] Transcription Bubble Model. https://www.google.com.ar/search?q=transcription+bubble+model\&tbm=isch\&tbo $=\mathrm{u} \&$ source $=$ univ\&sa $=$ X\&ved $=0$ ahUKEwj31 NalqIrYAhUGDpAKHTc3B5UQsAQIL g\&biw=1366\&bih=662\#im

[53] Gariaev, P.P. http://wavegenetics.org/es/issledovania

[54] P-53 Gen. https://es.wikipedia.org/wiki/P53

[55] Lingvistiko-Volonovoj Principles. http://wavegenetics.org/es/issledovania

[56] Pang, X.F., Chen, S., Wang, X. and Zhong, L. (2016) Influences of Electromagnetic Energy on Bio-Energy Transport through Protein Molecules in Living Systems and Its Experimental Evidence. International Journal of Molecular Sciences, 17, 20. https://doi.org/10.3390/ijms17081130 
[57] Pang, X.F. (2001) Vibrational Energy-Spectra of Protein Molecules and Non-Thermally Biological Effect of Infrared Light. 25th International Conference on Infrared and Millimeter Waves, Beijing, 12-15 September 2000, 298.

[58] Christiansen, L. and Scott, A.C. (1990) Davydov's Soliton Revisited: Self-Trapping of Vibrational Energy in Proteins. Nato Science Series B, Vol. 243, Springer Science + Business Media, New York, 121. https://doi.org/10.1007/978-1-4757-9948-4

[59] Christiansen, L. and Scott, A.C. (1985) (1990) Davydov's Soliton Revisited: Self-Trapping of Vibrational Energy in Proteins. Nato Science Series B, Vol. 243, Springer Science + Business Media, New York, 143. https://doi.org/10.1007/978-1-4757-9948-4

[60] Davydov, A.S. (1985) Solitons in Molecular Systems. D. Reidel Publishing, Dordrecht.

[61] Pang, X.F. (2001) Vibrational Energy-Spectra of Protein Molecules and Non-Thermally Biological Effect of Infrared Light. 25th International Conference on Infrared and Millimeter Waves, Beijing, 12-15 September 2000, 299.

[62] Plasmoids: Source: Energy Storage: Compression and Switching. Book 84, 80, Code BOS 42.

[63] Hashimoto's Theory: Source: Energy Storage: Compression and Switching. Book 84, 79-80, Code BOS 42.

[64] Adamski, A.G. (2016) The Importance of Movement, Solitons and Coherent Light in the Development of Mental Processes. Journal of Advanced Neuroscience Research, 3, 27. https://doi.org/10.15379/2409-3564.2016.03.01.04

[65] Gariaev, P.P. http://wavegenetics.org/es/issledovania/model-geneticheskogo-koda/15/

[66] Briggs, J. and Peat, F.D. (1994) Espejo y Reflejo: Del caos al orden. Editorial Gedisa, Segunda Edición, Barcelona, Chapter 4, 126.

[67] Briggs, J. and Peat, F.D. (1994) Espejo y Reflejo: Del caos al orden. Editorial Gedisa, Segunda Edición, Barcelona, Chapter 4, 128.

[68] Cruz, Y. and Fayad, R. (2011) Transporte de solitones en los MT: Un modelo clásico. Revista de la Facultad de Medicina, 19, No. 1.

http://www.scielo.org.co/scielo.php?script=sci_arttext\&pid=S0121-52562011000100 $\underline{009}$

[69] Cruz, Y. (2011) Microtúbulos y terapia neural: Propuesta de una investigación promisoria. 12. http://www.bdigital.unal.edu.co/4277/1/598635.2011.pdf

[70] Pang, X.F. (2001) Vibrational Energy-Spectra of Protein Molecules and Non-Thermally Biological Effect of Infrared Light. Monography for the University of Electronic Science and Technology of Chengdu, Chengdu, 300.

[71] Pang, X.F. (2001) Vibrational Energy-Spectra of Protein Molecules and Non-Thermally Biological Effect of Infrared Light. Monography for the University of Electronic Science and Technology of Chengdu, Chengdu, 302.

[72] Kotsias, B.A. Instituto de Investigaciones Médicas Alfredo Lanari, Facultad de Medicina, Universidad de Buenos Aires. (In Spanish)

[73] Neural Action Potential Recorded by Hodgkin and Huxley in Their Experiments. https://www.google.com.ar/search?q=trabajo+de+Hodgkin+Eccles+y+Huxley

[74] Teoría de solitones en la transmisión nerviosa. Medicina, (Buenos Aires), Vol. 64. (In Spanish)

http://www.scielo.org.ar/scielo.php?script=sci_arttext\&pid=S0025-76802004000300 $\underline{017}$ 
[75] Trajectory of the Giant Axon on the Body of the Squid (Doryteuthis pealeii or L. forbesi) Image.

$\underline{\text { http://www.scielo.org.ar/scielo.php?script=sci_arttext\&pid=S0025-76802004000300 }}$ $\underline{017}$

[76] Eccles, J. Works in the Synapses of the Central Nervous System. http://www.nobelprize.org/nobel_prizes/medicine/laureates/1963/

[77] Hodgkin, A. (1992) Chance and Design. Cambridge University Press, Cambridge.

[78] Huxley, A. (1988) Prefatory Chapter: Muscular Contraction. Annual Review of Physiology, 50, 1-16. https://doi.org/10.1146/annurev.ph.50.030188.000245

[79] Kandel, E.R. (2000) Nobelprizelauréate. (In Spanish) https://jralonso.es/2015/12/01/el-axon-gigante-del-calamar/

[80] Ivanisevic, V.G. and Ivancevic, T.T. (2013) Sine-Gordon Solitons, Kinks and Breathers as Physical Models of Nonlinear Excitations in Living Cellular Structures. Journal of Geometry and Symmetry in Physics, 31, 24. https://doi.org/10.7546/jgsp-31-2013-1-56

[81] Ivanisevic, V.G. and Ivancevic, T.T. (2013) Sine-Gordon Solitons, Kinks and Breathers as Physical Models of Nonlinear Excitations in Living Cellular Structures. Journal of Geometry and Symmetry in Physics, 31, 26. https://doi.org/10.7546/jgsp-31-2013-1-56

[82] Chou, K.C. and Shen, H.B. (2009) Recent Advances in Developing Web-Servers for Predicting Protein Attributes. Natural Science, 2, 63-92. https://doi.org/10.4236/ns.2009.12011

[83] Lin, S.X. and Lapointe, J. (2013) Theoretical and Experimental Biology in One. Journal of Biomedical Science and Engineering, 6, 435. https://doi.org/10.4236/jbise.2013.64054

[84] Ivanisevic, V.G. and Ivancevic, T.T. (2013) Sine-Gordon Solitons, Kinks and Breathers as Physical Models of Nonlinear Excitations in Living Cellular Structures. Journal of Geometry and Symmetry in Physics, 31, 28-32. https://doi.org/10.7546/jgsp-31-2013-1-56

[85] Chou, K.C. and Chen, N.Y. (1977) The Biological Functions of Low-Frequency Phonons. Scientia Sinica, 20, 447-457.

[86] Inchauspe, A.A. (2015) Therapeutic Acupunctural Resonance. 3rd Traditional Conference and Exhibition on Traditional \& Alternative Medicines, 3-5 August 2015, Birmingham.

[87] Inchauspe, A.A. (2017) Is Traditional Chinese Medicine Definitely an Exact Science? Comparison between Five Elements' Theory and Euclid Regular Polyhedrons' Postulates. EC Dental Science, 11, 255-277.

[88] Briggs, J. and Peat, F.D. (1994) Espejo y Reflejo: Del caos al orden. Editorial Gedisa, Segunda Edición, Barcelona, Chapter 4, 129.

[89] Solitons Sequential Frequencies. http://www.met.reading.ac.uk/pplato2/h-flap/phys5_1.html

[90] Hameroff, S. (2007) The Brain Is Both Neural Computer and Quantum Computer. Cognitive Science, 31, 1035-1045. https://doi.org/10.1080/03640210701704004

[91] Sedlak, W. (1975) Dynamika Bioplazm Metabolizm. Kosmos, 24, 261-272.

[92] Varela, F., Thompson, E. and Rosch, E. (1992) The-Emodied-Mind: Cognitive Science and Human Experience. Ed. Gedisa, Barcelona, 115. (In Spanish) 